\title{
Idosos vivendo na comunidade e a satisfação com a própria saúde bucal
}

\author{
A population of elderly and their satisfaction with their oral health
}

AlexandreFávero Bulgarelli ${ }^{1}$

AmábileRodrigues Xavier $\mathrm{M}$ anço ${ }^{1}$

\footnotetext{
${ }^{1}$ Faculdade de Medicina de Ribeirão Preto, Universidade de São Paulo. Av. Bandeirantes 3.900 M onte Alegre. 14049-902 Ribeirão Preto SP. alefavbulg@yahoo.com
}

Abstract Thismixed method study ai med at evaluating the satisfaction of elderly with their oral health, observing associated variables, and analyzing what would be necessary for satisfying their expectations with respect to oral health. Data were gathered by means of household interviews with 261 elderly people (city of Ribeirão Preto), representing the whole population of elderly registered in a Family Health Unit. A semi-structured questionnaire was elaborated to obtain information on the variables sex, age, educational level, financial independence, number of teeth and denture wearing. Data analysis was based on associations and content analysis. $49,2 \%$ of the totally toothless were satisfied whereas most of those who were not satisfied $(73.9 \%)$ were young-elderly people (60-69). There was a statistically significant association between educational level and satisfaction $(p=0,009) ; 76,0 \%$ of the elderly with low educational level were dissatisfied with their own oral health. The dissatisfied el derly related different situations that would make them feel satisfied. The most representative complaint mentioned in the interviews referred to discomfort caused by the denture. It was concluded that most of the elderly were satisfied with their oral health however, the higher the age and lower the educational level, the lower was the number of dissatisfied el derly. Key words Elderly, Oral health, Satisfaction, Old age
Resumo 0 presente estudo, deabordagem qualiquantitativa, objetiva levantar a satisfação deidosos em relação à saúde bucal, observar variáveis associadas e aspectos necessários para se sentirem sati sfeitos com a saúdebucal. Foram aplicadas entrevistas domiciliares em 261 idosos (Ribeirão Preto/SP), os quaisrepresentavam a população deidosos cadastrados em um N úcleo de Saúde da Família. Foi elaborado um questionário semi-estruturado abordando as variáveis sexo, idade, escolaridade, autonomia financeira, quantidadededentes euso de prótese. A análise dos dados se baseou em associações estatísticas e análise de conteúdo. Observou-se que $49,2 \%$ dos desdentados totais estavam satisfeitos, sendo que $73,9 \%$ dos insatisfeitos eram idosos-jovens (60-69 anos). H ouve associação estatisticamente significante entre escolaridade e satisfação $(p=0,009)$, onde $76,0 \%$ dos idosos com baixa escolaridade relataram estar insatisfeitos com a própria saúde bucal. O s insatisfeitos relataram diversas situações em que se sentiriam satisfeitos. A situação mais representativa esteve associada às queixas relatadas no momento da entrevista, como solucionar o desconforto causado pelas próteses bucais. Concluiu-se que a maioria dosidosos estava satisfeita, porém quanto maior a idade emenor a escolaridade, menor foi o número de insatisfeitos.

Palavras-chave Idoso, Saúde bucal, Satisfação, Velhice 
Introdução

No Brasil, os idosos, em geral, apresentam elevado número de dentes perdidos e necessidade de uso de prótese. $\mathrm{Na}$ velhice, estes aspectos retratam a história pregressa, ou seja, a história vivida das condições de saúde, bem como o tipo de atenção à saúde bucal que os idosos recebe ram ao longo da vida. Traçando-se o perfil do cenário epidemiológico brasileiro a partir da dé cada de 80, tem-se que nos levantamentos em saúde bucal realizados em 1986 e 1996, havia um alto índice de edentulismo entre adultos eidosos. No ano de 2003, ano em que foi realizado o último levantamento em saúde bucal no Brasil, o quadro de edentulismo ainda foi considerado alarmante ${ }^{1}$. Frente à desanimadora realidade da condição de saúde bucal dos idosos brasileiros, estudos em saúde pública suscitam o levantamento de novas hipóteses, para que melhorias na atenção eassistência à saúde bucal lentamente mudem esta realidade?2.

Ao observar o processo evolutivo da saúde bucal das populações de países em desenvolvimento, como éo caso do Brasil, pode-se afirmar quea saúde bucal encontra-se precária ${ }^{3,4}$, porém lentamente vem melhorando a cada década ${ }^{5-7}$. $\mathrm{Na}$ atualidade brasileira, grandes esforços e me Ihorias na atenção e assistência à saúde bucal já são uma realidade ${ }^{8}$. Paralelamente às políticas públicas, desaúdee sociais, tem-seque a preocupação com a promoção ea atenção à saúde bucal na velhice aflora em um momento de transição demográfica, em que o número de idosos, bem como a expectativa de vida do brasileiro, vêm crescendo. Referidas situações proporcionam uma lenta mudança no perfil da população idosa brasileira, a qual está envelhecendo com ligeira melhora nas condições de saúde, proporcionando assim uma melhor qualidade de vida. N este contexto, a velhice tem despertado a atenção de estudos científicos na odontologia devido ao aumento da população idosa que demanda adequados serviços de saúde, novas preocupações em nível familiar e social, dentre outras. Em outras palavras, tem-se que a odontologia na atualidade é promotora da saúde na velhice.

0 envelhecimento é vivido de maneira diferente de indivíduo para indivíduo, de uma geração para outra e de uma sociedade para outra. Assim, estudos com idosos devem embasar-seem diferentes elementos intrínsecos ao processo de envelhecimento, os quais são diretamenteligados às características do indivíduo, da sociedade, da cultura edas próprias políticas públicas vigentes?
Profissionais de saúde se deparam com diferentes pessoas idosas, que vivenciam diferentes valores, conceitos e graus de satisfação com a própria saúde, os quais se baseiam na informação, nos conhecimentos adquiridos e modificados pela experiência prévia e pelas normas sociais eculturai $\varsigma^{10}$. D estaca-se, neste contexto, a necessidade de novos objetivos e diferentes caminhos a serem traçados com a relação de escuta entre idosos e profissionais da saúde. Cabe aqui ressaltar que uma boa saúde bucal, entendida como parte integrante da saúde geral da pessoa idosa, é norteadora de um envel hecimento saudável, assim como a realização de atividades físicas e cognitivas, manutenção de contatos sociais e a prevenção de doenças ${ }^{11}$.

M ediante os aspectos abordados, o presente estudo teve como objetivo levantar o grau de satisfação de idosos em relação a sua própria saúde bucal, e observar possíveis variáveis associadas. Objetivou-se, também, levantar para os casos de idosos insatisfeitos quais as necessidades que os tornariam satisfeitos com a saúde bucal.

\section{Método}

0 presente estudo optou pela abordagem qualiquantitativa ${ }^{12}$, utilizando associações estatísticas e análise de conteúdo, através de dados obtidos por entrevistas semi-estruturadas. Assim, foram entrevistados 261 idosos cadastrados em um Núcleo deSaúde da Família na cidade de Ribeirão Preto/SP, todos com 60 anos ou mais de idade, mental mente capacitados e fisicamente independentes. N estecontexto, lançou-semão deum instrumento - M ini ExamedeEstado M ental ${ }^{13}{ }_{-}$, para exclusão de idosos com incapacidade cognitiva. Foram utilizados os formulários (cadastros) das famílias para observação de possíveis dependências físicas como artrose crônica, seqüelas motoras de acidente vascular cerebral eoutras doenças crônicas que tornassem osidosos dependentes de familiares e/ou cuidadores. Os idosos com incapacidade cognitiva e dependência física não fizeram parte dos 261 idosos entrevistados.

Para a obtenção dos dados foi elaborado, para o presenteestudo, um questionário contendo questões objetivas abordando a temática a ser estudada. Foram trabal hadas as variáveis: sexo, escolaridade, idade, autonomia sobrea própria renda, quantidade de dentes e uso de prótese. Já a abordagem da satisfação foi levantada segundo questões de categorização e de classificação. U ma questão aberta observou a insatisfa- 
ção com a saúdebucal (Apêndice 1). Este instrumento foi aplicado verbalmente e as respostas obtidas foram escritas pelo pesquisador, por próprio punho, no questionário. Os referidos instrumentos, já preenchidos, foram arquivados e devidamente numerados de acordo com as entrevistas. Os dados foram digitados em máscara, utilizando-se o programa Epidata. Para estudar quantitativamente os dados, os mesmos receberam tratamento estatístico com análise descritiva de freqüência simples eforam observadas associações entre variáveis categorizadas deacordo com o teste Q ui-quadrado e o Teste Exato de Fischer. Foram utilizados os programas Epi-info e Stata, onde a hipótese de associação foi aceita frentevalor de menor ou igual a 0,05. Para trabalhar os dados qualitativamente, aplicou-se análise de conteúdo ${ }^{14}$, para estudo de categorias do discurso (análise categorial), na resposta da questão aberta. Vale ressaltar que foram agrupadas as respostas de todos os sujeitos e analisadas diversas perspectivas dos assuntos centrais.

0 presente estudo teve seu projeto submetido ao Comitêde Ética em Pesquisa do Centro de Saúde Escola da Faculdade de M edicina deRibeirão Preto da Universidade de São Paulo, obtendo aprovação para seu desenvolvimento. Todos os idosos participantes foram previamente orientados e concordaram por livre espontânea vontade em participar da pesquisa, assinando o Termo de Consentimento LivreeEsclarecido segundo resolução do Conselho N acional de Saúde. Nos casos de idosos não alfabetizados, a livre concordância se efetivou mediante a assinatura de um outro termo específico devidamente assinado por seu responsável.

\section{Resultadosediscussão}

Por se tratar de um estudo que se norteia em duas abordagens de análise dos dados, optou-se por uma apresentação e discussão dos resultados em duas etapas, proporcionando um meIhor caminho a ser percorrido no desfrutar da leitura.

\section{Análisequantitativa}

A população entrevistada foi composta de 261 indivíduos, sendo $65,5 \%$ mulheres e $34,5 \%$ homens. Esta situação, na qual o sexo feminino é mais representativo na população de estudo, também foi observada por diversos autores ${ }^{4,7,15,16}$. A idade variou de 60 a 91 anos, sendo que $46,7 \%$ possuíam de 60 a 69 anos, 43,3\%, de 70 a 79 e apenas $10,0 \%$ possuíam 80 anos ou mais. Do total dos idosos, $6,1 \%$ relataram possuir todos os dentes e $47,8 \%$ eram desdentados totai s eusuários depróteses dentárias. Dado semelhantepara o edentulismo em população deidosos (55,74\%) foi relatado na literatura em estudo sobre necessidades de tratamentos odontológicos para pessoas idosas 4 . Elevado índice de edentulismo $(52,1 \%)$ também foi encontrado na cidade de Recife/PE em idosos não institucionalizados ${ }^{17}$. Um outro estudo, realizado na década de 90 na cidade de São Paulo/SP, já apontava os altos índices de edentulismo em idosos necessitando de tratamentos protéticos ${ }^{18}$. No presente estudo, a grande maioria $(62,0 \%)$ possuía quatro anos de estudo eapenas $17,6 \%$ possuíam autonomia sob sua própria renda. A autonomia do idoso em relação à própria renda não foi encontrada na literatura como observada no presente estudo; porém, estudos sobre condições socioeconômicas na saúde bucal de idosos mostram que $13,34 \%$ não possuíam renda e $76,67 \%$ apresentavam baixo nível de escolaridade ${ }^{19}$.

Do total da população entrevistada, 170 sujeitos, o que corresponde a $65,1 \%$ da população estudada, alegaram estar satisfeitos e/ou muito satisfeitos em relação à própria saúde bucal. A penas dois sujeitos se demonstraram indiferentes em relação ao questionamento. Quando os idosos foram questionados sobre satisfação em relação a sua condição da saúde bucal, observouse que, independente da quantidade de dentes relatada, a resposta mais expressiva foi a satisfação (Tabela 1). Estes dados assemelham-se a estudos que mostram que a satisfação com a saúde bucal é alta quando o sujeito do estudo épessoa idosa ${ }^{20,21}$.

No presente estudo, com o avançar da idade, os idosos relataram maior grau de satisfação com saúde bucal; porém, não foi observada associação estatisticamente significante entre satisfação e a variável sexo $(p=0,583)$ (Tabela 2). Pôde-se afirmar que mulheres e homens tiveram pontos de vista semel hantes mediante à satisfação com a saúde bucal. Foi possível observar que, quanto maior a idade, mais expressiva foi a satisfação com a saúde bucal, e houve associação significante entre idade e satisfação, como mostrado na Tabela 2.

Ao analisar a satisfação em relação aos anos de estudo, observou-se que os idosos com menor escolaridade estavam mais satisfeitos com a saúde bucal. Similarmente ao presente achado, idosos entrevistados em diferentes trabalhos re- 
latados na literatura retrataram a saúde bucal como um aspecto positivo da sua saúde, em outras palavras, relataram estar satisfeitos com a saúde bucal ${ }^{22-24}$. A satisfação do paciente que é submetido a algum cuidado odontológico é um processo complexo, social e de relação pacientedentista que resulta de experiências com o tratamento anteriormente vivido ${ }^{25}$.

H ouveassociação estatisticamente significante entre quantidade de dentes relatada e satisfação $(p=0,001)$ (Tabela 2), e assim observou-se que, dentre os satisfeitos, 49,2\% eram desdentados totais, $23,1 \%$ eram parcialmente dentadose $27,7 \%$ eram totalmente dentados, como mostra a Tabela 1. A associação entre a satisfação e a quantidade de dentes foi encontrada também na literatura em estudo de Carvalho ${ }^{26}$, que mostrou haver associação entre a satisfação com a saúde bucal e o número de dentes; entretanto, esta situação foi superestimada, ou seja, condições clínicas ruins, mas satisfação com a saúde bucal. Outros estudos apontam que a satisfação esteve associada a variáveis socioeconômicas, aparência dos dentes e próteses, e habilidade para falar claramente e, nestes casos, desdentados totais e indivíduos de baixa renda relataram maior insa- tisfação com a saúde bucal ${ }^{20,21}$. Na literatura, encontra-se relatada a insatisfação dos idosos desdentados, sempre associada à condição dos aparelhos protéticos devido a próteses soltas, gastas e fraturadas, 0 que dificulta a mastigação ${ }^{27}$.

Ao abordar a situação financeira, observouse que a autonomia do idoso sob sua própria renda não esteve associada à satisfação com a saúde bucal $(p=0,102)$ (Tabela 2). Não foi objetivo do presente estudo o levantamento da variável renda de acordo com indicadores socioeconômicos largamenteutilizados em pesquisas populacionais, visto que a situação em relação à renda do indivíduo idoso é um fator muito delicado a ser mencionado e trabalhado, pois certos idosos por muitas vezes são beneficiários de alguma renda, todavia não têm autonomia sobreela. Seu dinheiro émuitas vezes utilizado em benefício de outros indivíduos da família e/ou grupo em que vive ${ }^{28}$. Pode-se dizer que, no presente estudo, a autonomia sobre a renda não influenciou no grau de satisfação com a saúde bucal, pois não houve associação estatisticamente significante $(p=0,102)$, como mostrado na Tabela 2.

Ao observar estudos relacionados à saúde bucal de idosos, alguns autores mostraram a

Tabela 1. Distribuição do grau de satisfação com a saúde bucal. Idosos cadastrados em um núcleo de saúde da família. Ribeirão Preto/SP, 2005.

\begin{tabular}{|c|c|c|c|c|c|c|c|c|}
\hline \multirow[t]{2}{*}{ Variável } & \multicolumn{2}{|c|}{ Indiferente } & \multicolumn{2}{|c|}{ Insatisfeito } & \multicolumn{2}{|c|}{ Satisfeito } & \multicolumn{2}{|c|}{ Muito satisfeito } \\
\hline & $\mathrm{n}$ & $\%$ & $\mathrm{n}$ & $\%$ & $\mathrm{n}$ & $\%$ & $\mathrm{n}$ & $\%$ \\
\hline \multicolumn{9}{|l|}{ Sexo } \\
\hline Masculino & 0 & 0,0 & 45 & 48,9 & 73 & 52,8 & 9 & 31,1 \\
\hline Feminino & 2 & 0,0 & 47 & 51,1 & 65 & 47,2 & 20 & 68,9 \\
\hline \multicolumn{9}{|l|}{ Idade } \\
\hline $60-69$ & 0 & 0,0 & 68 & 73,9 & 37 & 26,8 & 6 & 20,8 \\
\hline $70-79$ & 1 & 50,0 & 14 & 15,2 & 45 & 32,6 & 11 & 37,9 \\
\hline 80 e + & 1 & 50,0 & 10 & 10,9 & 56 & 40,6 & 12 & 41,3 \\
\hline \multicolumn{9}{|l|}{ Escolaridade } \\
\hline Até 4 anos & 2 & 100,0 & 3 & 3,4 & 59 & 42,7 & 10 & 34,4 \\
\hline 5 a 9 anos & 0 & 0,0 & 19 & 20,6 & 41 & 29,7 & 17 & 58,6 \\
\hline 10 ou mais & 0 & 0,0 & 70 & 76,0 & 38 & 27,6 & 2 & 7,0 \\
\hline \multicolumn{9}{|c|}{ Quantidade de dentes } \\
\hline Edêntulo & 2 & 100,0 & 5 & 5,5 & 68 & 49,2 & 9 & 31,0 \\
\hline Parc. dent. & 0 & 0,0 & 33 & 35,8 & 32 & 23,1 & 7 & 24,2 \\
\hline Dentado & 0 & 0,0 & 54 & 58,7 & 38 & 27,7 & 13 & 44,8 \\
\hline \multicolumn{9}{|c|}{ Autonomia sob própria renda } \\
\hline Sim & 0 & 0,0 & 41 & 44,6 & 79 & 57,2 & 17 & 58,6 \\
\hline Não & 2 & 100,0 & 51 & 55,4 & 59 & 42,8 & 12 & 41,4 \\
\hline
\end{tabular}


Tabela 2. Associação das variáveis estudadas em relação a satisfação com a saúde bucal. I dosos cadastrados em um núcleo de saúde da família. Ribeirão Preto/SP, 2005.

\begin{tabular}{|c|c|}
\hline $\begin{array}{l}\text { Satisfação com a saúde bucal } \\
\text { Variáveis estudadas }\end{array}$ & $\begin{array}{l}\text { Valor } \\
\text { de } p\end{array}$ \\
\hline Sexo & 0,583 \\
\hline Idade & 0,018 \\
\hline Escolaridade & 0,009 \\
\hline Quantidade de dentes & 0,001 \\
\hline U so de prótese total & 0,013 \\
\hline Situação financeira & 0,102 \\
\hline
\end{tabular}

ausência de uma padronização da classificação quanto à idade, bem como uma apresentação de resultados quando se estuda edentulismo, uso e necessidade deprótese em uma população deidosos. Este aspecto dificulta a comparação de achados entre diversos outros trabalhos científi$\cos ^{28,29}$. Neste raciocínio, há a necessidade de novos estudos padronizados e que levantem novos contextos e variáveis para se criar novas hipóteses a serem discutidas². Cabe ressaltar que, historicamente, as variáveis associadas à saúde bucal têm sido avaliadas por parâmetros clínicos e epidemiológicos. Assim, não se consideram de maneira efetiva as dimensões sociais da saúde bucal. Segundo o mesmo autor, é esta dimensão social que reflete os problemas na qualidade de vida das pessoas idosas ${ }^{30}$, e os valores, cuidados e satisfação com a saúde bucal.

\section{Análise qualitativa}

Dentre todos os idosos participantes da pesquisa, 34,1\% (89 idosos) alegaram insatisfação com a própria saúde bucal. Estes mesmos idososforam questionados sobretal assunto (A pêndice 1). 0 estudo se preocupou em observar 0 que levaria o idoso a se sentir satisfeito com a saúde bucal e, por saturação dos dados, a amostra estudada foi de 17 idosos. Ao se observar um texto que expressa comunicação de diferentes sujeitos sobre um mesmo assunto, foi possível conhecer aquilo que estava por trás das palavras. Assim, a análise de conteúdo, sendo um conjunto de técnicas de análise das comunicações descrevendo o conteúdo das mensagens, vem embasar o presente estudo. Dentre as técnicas da análise de conteúdo, utilizou-se a análise categorial, a qual desmembra os textos das mensagens em categorias e estabelece 0 agrupamento por significados semânticos ${ }^{14,31}$. Foi possível observar diferentes temas dentre as mensagens obtidas com as respostas e, durante o processo de análise, valorizou-se a evocação dos temas.

A satisfação humana é um fato rodeado de complexidades que envolvem conceitos, valores, expectativas, experiências passadas e estilos de vida. Diante disso, estudos científicos buscam as fontes de uma possível insatisfação ${ }^{32}$. Baseado neste paradigma, o presente estudo tentou buscar e trabalhar a insatisfação.

Foram identificados, através da ocorrência de quatro temas, os quais emergiram da leitura do material, as categorias que expressavam as unidades temáticas referentes à necessidade para se tornarem satisfeitos com a saúde bucal. Estas quatro categorias foram distribuídas em grupos. o Grupo 1, referente aos idosos que gostariam de melhorar sua auto-imagem para se sentirem satisfeitos com sua própria saúde bucal. 0 Grupo 2, referente aos idosos que declararam a necessidade de utilização de novos (modernos) recursos dereabilitação protética. 0 Grupo 3, referente aos idosos que al egaram a necessidade de melhores condições socioeconômicas para cuidar da saúde bucal e se sentirem satisfeitos; e o Grupo 4 declarou que para se sentir satisfeito com a saúde bucal necessitava apenas sanar a queixa atual (Quadro 1).

As necessidades para se tornarem satisfeitos com a saúde bucal estão relacionadas aos aspectos que estabelecem uma satisfatória qualidade de vida destes idosos. Portanto, a saúde bucal vivida por estes idosos mostra suas necessidades e satisfações. Estudos apontam que a imagem em relação à saúde bucal afeta a qualidade de vida de alguns idosos ${ }^{33,34}$. Assim, a aparência da boca como um todo reflete uma melhor qualidade de vida. Leao e Sheiham ${ }^{35}$ sugeriram que a satisfação com a saúde bucal, bem como a situação social e psicológica, devem ser analisadas quando questões como "necessidades" são questionadas aos idosos. Estefato serviu de base para a seleção dos idosos entrevistados no presente estudo, visto que idosos com incapacidade cognitiva foram excluídos da população de estudo. Com a lenta, porém contínua melhora nas condições da saúde bucal da população brasileira ${ }^{6}$, existe um maior número de dentes nas populações com idades mais avançadas e conseqüentemente novas preocupações vão surgindo para esta parcela da população. 
Quadro 1. Categorização da evocação do tema. Idosos cadastrados em um núcleo de saúde da família. Ribeirão Preto/SP, 2005

\begin{tabular}{|c|c|c|c|c|}
\hline Grupos & Categorias & Evocação & $n$ & $\%$ \\
\hline Grupo 1 & $\begin{array}{c}\text { Melhorar a } \\
\text { aparência/imagem }\end{array}$ & $\begin{array}{l}\text { Lábios } \\
\text { Perfil do rosto } \\
\text { Estética dos dentes }\end{array}$ & $\begin{array}{l}6 \\
3 \\
9\end{array}$ & $\begin{array}{l}33,3 \\
16,7 \\
50,0\end{array}$ \\
\hline Grupo 2 & $\begin{array}{c}\text { Necessidade de utilização recursos } \\
\text { modernos / atuais }\end{array}$ & $\begin{array}{l}\text { Implantes } \\
\text { Reabilitações fixas }\end{array}$ & $\begin{array}{l}15 \\
10\end{array}$ & $\begin{array}{l}60,0 \\
40,0\end{array}$ \\
\hline Grupo 3 & $\begin{array}{c}\text { Melhorar condições socioeconômicas } \\
\text { para cuidar da saúde bucal }\end{array}$ & $\begin{array}{l}\text { Possuir dinheiro } \\
\text { Possuir tempo }\end{array}$ & $\begin{array}{l}2 \\
1\end{array}$ & $\begin{array}{l}66,6 \\
33,4\end{array}$ \\
\hline Grupo 4 & $\begin{array}{l}\text { Sanar apenas } \\
\text { a queixa atual }\end{array}$ & $\begin{array}{l}\text { Trocar prótese } \\
\text { Fazer limpeza } \\
\text { Curar a gengiva } \\
\text { Sanar a dor }\end{array}$ & $\begin{array}{r}27 \\
3 \\
10 \\
3\end{array}$ & $\begin{array}{r}62,9 \\
6,8 \\
23,5 \\
6,8\end{array}$ \\
\hline
\end{tabular}

No presente estudo, observou-se que alguns idosos se preocupavam com a imagem que mostravam para o mundo social, e esta imagem encontrou-se diretamente relacionada à estética. A ausência de dentes está diretamente ligada à insatisfação estética, o que acaba por gerar estímulos negativos que envolvem as relações sociais ${ }^{36}$. Em alguns casos, a característica fisiológica que surge devido à perda da dimensão vertical acarretada pela ausência dos dentes ea não utilização de próteses dentárias reabilitadoras, o que aproxima a distância fisiológica entre o ponto mais extremo do queixo e a ponta do nariz, também foi relatado como um motivo de insatisfação. Paralelo a estes aspectos de insatisfação, observou-se que a aparência dos lábios (lábios sem tonicidade muscular), a qual reflete a imagem do "ser idoso", "estar velho" e conseqüentemente mostrar as marcas do tempo, foi relatada por alguns sujeitos como uma situação para ser vivenciada no futuro. Isto mostra a despreocupação com o tempo ${ }^{37}$. Leao e Sheiham ${ }^{35}$ relataram que a insatisfação com a aparência da boca estava associada com a perda dentária e com a perda da função mastigatória nos idosos. Estudo realizado na cidade de Goiânia/GO observou que os idosos se referem à saúde bucal como uma preocupação estética, dentre outras. Este aspecto mostra a preocupação com a imagem, aparência do corpo no mundo social ${ }^{7}, \mathrm{e}$ este fato está associado à satisfação com a saúde bucal, como no presenteestudo. Pôde-secaracterizar o Grupo 1 (Quadro 1) como sendo referen- te à vaidade estética, o que mostra que alguns idosos se preocupam e vivem este sentimento em relação à saúde bucal.

Sabe-se que os idosos, na atualidade, estão vivendo uma nova realidade, na qual avanços na medicina, bem como em outras áreas da saúde, estão proporcionando novos desafios e a integralidade e a humanização dos serviços de saúde. Estes idosos estão buscando novas informações e conseqüentementeestão solicitando novos recursos de reabilitação dentária. M uitos já deixam de lado a idéia de usar "dentadura" epartem em busca de recursos atuais, como os relatados no presente estudo (implantes). Estas evocações, representadas por sujeitos do Grupo 2 (Quadro 1), mostram que, para a população estudada, a informação foi um fato presente eque na sociedade este contexto vem proporcionar buscas por novos tratamentos, em sujeitos que em um passado recente consentiam com extrações múltiplas eutilização de "dentaduras". Tepper et al. ${ }^{38}$ relataram, em trabalho sobre implantes dentários osteointegrados, que $25 \%$ dos idosos entrevistados conheciam alguma pessoa que havia feito implante e estavam satisfeitas com este meio de adequar a saúde bucal. Este fato, por si só, reflete o acesso à informação para o cuidado com a saúde bucal, que lentamente está se tornando uma realidade na promoção de saúde bucal.

Alguns sujeitos do estudo associaram a necessidade para setornarem satisfeitos com a saúde bucal diretamente com a situação socioeconô- 
mica. Como exemplo, um idoso entrevistado afirmou que precisaria melhorar sua situação financeira, e outro relatou que seria necessário mais tempo disponível em suavida. O u seja, com tempo e dinheiro passariam a se preocupar com a saúde bucal e conseqüentementetal fato Ihes proporcionaria satisfação com saúde da boca.

A situação socioeconômica, juntamente com a situação da saúde geral do idoso, representam fatores que estão diretamente associados à percepção da saúde bucal. N ão é apenas a situação clínica dos dentes queinfluencia a satisfação com a saúde bucal, esim o contexto geral implícito na vida do idoso ${ }^{39}$. A barreira socioeconômicaéuma situação que conduz à insatisfação com a saúde bucal e estefato sempreesteve associado à crença dos altos custos com o serviço odontológico, 0 que impede 0 idoso de cuidar da saúde bucal ${ }^{40}$. Ou seja, a satisfação com a saúde bucal esteve representada por alguns sujeitos da pesquisa pela dificuldade do acesso ao dentista. Alguns idosos não estão insatisfeitos com a sua real condição fisiológica e/ou patológica do sistema bucal, mas sim com fatores do mundo social que impossibilitam o cuidado, bem como o fato de não terem acesso ao referido cuidado.

Dentre todos os idosos insatisfeitos, muitos apenas relataram insatisfação com a condição da saúde bucal no momento da entrevista e situações de incômodo em que a estética o uso de recursos mais modernos e a situação socioeconômica não foram representativos de insatisfação. Tais sujeitos não se preocupavam com o futuro, tendo sido observado que suas insatisfações estavam associadas à preocupação em sanar problemas referentes à queixa atual. Tais queixas mostravam os problemas agudos os quais estavam sendo vivenciados no momento da entrevista. Os idosos relataram que precisariam apenas cuidar de problemas na gengiva, sanar dores e refazer trabalhos protéticos. Ressalta-se que, na literatura, as queixas relatadas por idosos, na grande maioria, estão associadas a problemas com próteses dentárias ${ }^{41}$. N este âmbito, cabe aqui resgatar Santos ${ }^{36}$, o qual enfatiza que alguns idosos possuíam uma despreocupação com o tempo futuro, o que lhes direciona a se preocupar com o tempo presente.

\section{Consideraçõesfinais}

N a população estudada, foi possível observar que os idosos estavam informados e valorizando a saúde bucal, bem como se preocupando e buscando informações sobre o "cuidar em saúde". Com o avançar da idade, acredita-se que devido à despreocupação com o tempo, os idosos se tornam mais tolerantes com os problemas da cavidade bucal que surgem ao longo da velhice e, neste contexto, relataram maior satisfação com a saúde bucal. Q uanto menor o grau de escolaridade e maior a idade, maior foi a sati sfação com a saúde bucal relatada na presente pesquisa. Em outro perfil, a autonomia sob a renda não esteve associada à satisfação com a saúde bucal; porém, tal fato em alguns casos foi motivo de insatisfação com a saúde bucal, pois esteve relacionado ao difícil acesso ao cuidado (barreira econômica), o que gerou indignação e insatisfação. Fato interessante foi relatado por alguns idosos os quais associaram a imagem, aparência da boca que mostram para a sociedade, como sendo um motivo de insatisfação e, por outro lado, houve aqueles idosos que possuíam uma insatisfação momentânea, resultado de uma queixa presente no desenvolver da pesquisa. Dentre tais dados, considera-se que a busca por informação, preocupações estéticas, diferentes situações socioeconômicas e queixas em saúde bucal são fatos presentes e vivenciados nesta população idosa que, à semelhança da população idosa brasileira, está iniciando uma lenta mudança de perfil devido ao novo paradigma da promoção da saúde bucal.

\section{Colaboradores}

AF Bulgarelli trabal hou na concepção, coleta dos dados, análise estatística e de conteúdo, bem como na redação, e ARX $M$ anço trabalhou na concepção, análise estatística e de conteúdo, bem como na redação e revisão final do estudo. 
Apêndice 1

Faculdade de M edicina de Ribeirão Preto

Universidade de São Paulo

INSTRUM ENTO*: Satisfação em relação saúde bucal. Ribeirão Preto/SP.

Questionário número:

Data da entrevista:

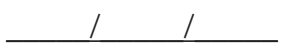

IDENTIFICAÇÃO

Data de nascimento: Idade em anos: Sexo: ( ) M ( ) F

Escolaridade

( ) Não alfabetizado ( ) Primário ( ) 1o grau ( ) 2o grau ( ) Grau superior

1 - Com quem o sr(a) mora nesta casa?

( ) só ( ) com familiares ( ) com companheiro/(a) ( ) com amigos

2 - 0 sr(a) tem/recebe algum dinheiro que fica sob o seu controle/cuidado? （ ) Sim （） Não

3 - Quanto ao número de dentes o sr(a) :

( ) Não tem dente algum (desdentado total)

( ) Tem alguns dentes (elementos naturais e/ou reabilitações fixas)

( ) Tem todos os dentes (elementos naturais e/ou reabilitações fixas) (pular para a questão 5)

4 - O sr(a) tem "dentadura" ( ) Sim ( ) Não- e/ou "ponte móvel"? ( ) Sim （） Não

5 - De acordo com a saúde da sua boca o sr(a) está:

\begin{tabular}{|c|c|c|c|}
\hline 0 & 1 & 2 & 3 \\
\hline
\end{tabular}

5a- Em caso de insatisfação. 0 que precisaria pra ficar satisfeito? **

* Fragmento do instrumento da pesquisa de mestrado intitulada: Saúde bucal em I dosos. Q ueixas relatadas, Ribeirão Preto (SP). Realizada na Faculdade de M edicina de Ribeirão Preto da U niversidade de São Paulo.

** Questão norteadora. 


\section{Referências}

1. Roncalli AG. Epidemiologia e saúde bucal coletiva: um caminhar compartilhado. Cien Saude Colet 2006; 11(1):105-114.

2. Nadanovsky $P$ [editorial]. $O$ aumento da produção científica odontológica brasileira na saúde pública. Cad Saúde Pública 2006; 22(5).

3. Moreira RS, Nico LS, Tomita NE, Ruiz T. A saúde bucal do idoso brasileiro: revisão sistemática sobre o quadro epidemiológico e acesso aos serviços de saúde bucal. Cad Saúde Pública 2005; 21(6):1665-1675.

4. Tibério D, Santos MTBR, Ramos LR. Estado periodontal e necessidade de tratamento em idosos. Rev. Assoc. Paul. Cir. Dent 2005; 59(1):69-72.

5. Brasil. Ministério da Saúde. Divisão Nacional de Saúde Bucal. Levantamento em saúde bucal - Brasil, zona urbana, 1986. Brasília: M inistério da Saúde; 1988.

6. Brasil. M inistério da Saúde. Coordenação Nacional de Saúde Bucal. Projeto SB Brasil 2003: condições de saúde bucal da população brasileira 2002-2003. Brasília: M inistério da Saúde; 2004.

7. Reis SCB, Marcelo VC. Saúde bucal na velhice: percepção dos idosos, Goiânia, 2005. Cien Saude Colet 2006; 11(1):191-199.

8. Brasil. M inistério da Saúde. Plano Nacional de Saúde Bucal. Diretrizes da política nacional de saúde bucal. Brasília: Ministério da Saúde; 2004.

9. Uchôa E, Firmo JA, Lima-Costa MFF. Envelhecimento e Saúde: experiência e construção cultural. In: M inayo M CS, Carlos EAC, organizadores. Antropologia, saúde e envelhecimento. Rio de Janeiro: Fiocruz; 2002. p. 25-36.

10. Portillo JC, Paes AM. Autopercepção da qualidade de vida relativa à saúde bucal. Rev. Bras Odont Saúde Col 2000; 1(1):75-88.

11. Kiyak HA. Successful aging: implication for oral health. J. Public. H ealth Dent 2000; 60(4):276-281.

12. Sandelowski M. Combining qualitative and quantitative sampling, data collection, and analysis techniques in mixed-method studies. Res $\mathrm{N}$ urs $\mathrm{H}$ ealth 2000; 23(3):246-255.

13. Seabra MLV, Concílio GV, Villares JB, Carlini EA Avaliação do teste "M ini-Mental State" em voluntários e pacientes brasileiros. Rev. ABP-APAL 1990; 12(1):1-7.

14. Bardin L. Análise de conteúdo. Lisboa: Edições 70; 1995.

15. M eneghim MC, Saliba NA. Condições de saúde bucal da população idosa de Piracicaba - SP: 1998. RPG Rev. Pós Grad 2000; 7(1):7-13.

16. Oliveira JA, Ribeiro EDP, Bonachela WC, Capelozza ALA. Perfil do paciente odontogeriátrico da Faculdade de Odontologia de Bauru - USP. PCL 2002; 4(17):71-79.

17. Caldas Junior AF, Figueiredo ACL, Soriano EP, Sousa EHA, M elo JBG, Vilela AS. Prevalência de cárie e edentulismo em idosos de Recife - Pernambuco -

18. Brasil. Rev. bras. ciênc. saúde 2002; 6(2):113-122. Rosa AGF, Fernandez RAC, Pinto VG, Ramos LR. Condições de saúde bucal em pessoas de 60 anos ou mais no Município de São Paulo (Brasil). Rev. Saúde Pública 1992; 26(3):155-160.
19. Peres SHCS, Peres AS. Determinantes das condições socioeconômicas na saúde bucal da terceira idade. RPG Rev. Pós Grad. 2003; 10(4):369-375.

20. Jokovic A, Locker D. Dissatisfaction with oral health status in an adult population. J Public Health Dent 1997; 57(1):40-47.

21. Locker D, Matear D, Stephens M, Jokovic A. Oral health-related quality of life of a population of medically compromised elderly people. Community Dent Health 2002; 19(2):90-97.

22. Matthias RE, Atchison KA, Lubben JE, De Jong $F$, Schweitzer SO. Factors affecting self-ratings of oral health. J Public Health Dentistry 1995, 55: 197-204.

23. Cruz GD, Galvis DL, Kim M, Le-Geros RZ, Barrow SY, Tavares M, Bachiman R. Self-perceived oral health among three subgroups of Asian-Americans in New York city: a preliminary study. Community Dent Oral Epidemiol 2001; 29:99-106.

24. Ekanayke L, Perera I. Factors associated with perceived oral health status in older individuals. Inter Dent J 2005; 55:31-37.

25. Newsome PRH, Wright GH. A review of patient satisfaction: Dental patient satisfaction, an appraisal of recent literature. British Dent J 1999; 186(4):166-170.

26. Carvalho IM M. Avaliação sócio-odontológica de 300 pessoas idosas de Bauru/SP [tese]. Bauru (SP): Faculdade de Odontologia de Bauru, Universidade de São Paulo; 2000.

27. Braga SRS, Telarolli Júnior R, Braga AS, Catirse ABCEB. Avaliação das condições e satisfação com as próteses em idosos da região central do estado de São Paulo (Brasil). Rev. O dontol. UNESP 2002; 31(1):39-48

28. Bulgarelli AF, M anço ARX. Saúde, idosos e autonomia financeira. Anais II Seminário de Saúde Coletiva. Desigualdades em saúde, uma abordagem interdisciplinar; 2004 Nov 26-27; Florianópolis, Brasil.

29. Colussi CF, Freitas SFT. Aspectos epidemiológicos da saúde bucal do idoso no Brasil. Cad Saúde Pública 2002; 18(5):1313-1320.

30. Chen MS, Hunter P. Oral health and quality of life in New Zealand: A social perspective. Social Science and M edicine 1996; 43:1213-1222.

31. Patton M Q. Qualitative evaluation and research methods. Thousand Oaks: Sage Publication; 1990.

32. U sual $A B$, Araujo AA, Diniz FVM, Drumond MM. $N$ ecessidade sentida e observada: suas influencias na satisfação de pacientes e profissionais. Arq O dontol 2006; 42(1):65-80.

33. Hollister MC, Weintraub JA. The association of the oral status with systemic health, quality of life and economics productivity. J. Dent Educ 1993; 57:901912.

34. Slade GD, Spencer AJ. Development and evaluation of the oral health impact profile. Com Dent Res 1994; (11):3-11.

35. Leao A, Sheiham A. Relation between clinical dental status and subjective impacts on daily living. J Dent Res 1995; 74(7):1408-1413.

36. Almeida MEL. Envelhecimento e bucalidade: suas múltiplas dimensões [tese]. Araçatuba (SP): Faculdade de Odontologia; 2003. 
37. Santos WT. 0 olhar do idoso sobre sua própria saúde [tese]. São Paulo (SP): Escola de Saúde Pública; 2001.

38. Tepper G, Haas R, M ailath G, Teller C, Bernhart T, Monov $G$, Watzek $G$. Representative marketing-oriented study on implants in the Austrian population. Implant acceptance, patient-perceived cost and patient satisfaction. Clin. Oral Implant Res 2003; 14:634-642.

39. Gilbert $G H, H$ eft $M W$, Duncan RP, Ringelberg $M L$. Perceived need for dental care in dentate older adults. Int Dent J 1994; 44:145-152.

40. Nakazono TT, Davidson PL, Andersen RM. Oral health beliefs in diverse populations. Adv Dent Res 1997; 11(2):235-244.

41. Bulgarelli $A F, M$ anço $A R X$. Os idosos se queixam de suas próteses dentárias? Rev FOA 2006; 8(1):26-29.

Artigo apresentado em 26/06/2007

Aprovado em 07/03/2007

Versão final apresentada em 05/07/2007 